\title{
Enriching Musical Interaction on Tactile Feedback Surfaces with Programmable Friction
}

\author{
Farzan Kalantari, Florent Berthaut and Laurent Grisoni \\ University of Lille, CNRS, INRIA Lille, France \\ farzan.kalantari@inria.fr, florent.berthaut@univ-lille1.fr, \\ laurent.grisoni@univ-lille1.fr
}

\begin{abstract}
In the recent years, a great interest has emerged to utilize tactile interfaces for musical interactions. These interfaces can be enhanced with tactile feedback on the user's fingertip through various technologies, including programmable friction techniques. In this study, we have used a qualitative approach to investigate the potential influence of these tactile feedback interfaces on user's musical interaction. We have experimented three different mappings between the sound parameters and the tactile feedback in order to study the users' experiences of a given task. Our preliminary findings suggest that friction-based tactile feedback is a useful tool to enrich musical interactions and learning.
\end{abstract}

Keywords: Tactile musical interface; Haptic rendering; Frequency modulation; Programmable Friction; Musical perception; User experience

\section{Introduction}

Touch interactions with tactile interfaces such as smart-phones and tablets, have become more and more ubiquitous in our daily life. However, there is still a lack of dynamic haptic feedback on these tactile interfaces to the user's finger. Buxton et al. [1] has shown that flat touchscreens need haptic feedback in order to ease the users' various interaction tasks, to enhance the efficiency of the interfaces and to increase the feeling of realism in visual environments. Accordingly, numerous studies have investigated the use of tactile interfaces with haptic feedback for sound synthesis and musical productions $[2-5,7,8]$.

Tactile feedback technologies are mostly based on the modulation of the friction between the user's fingertip and the tactile interface. Different types of vibrotactile actuators can be used for tactile rendering [9]. "Electrovibration" [10, 11] and "electroadhesion" [12] technologies, which both increase the friction between the user's fingertip and the tactile interface by applying the electrostatic force. The first technology by means of a voltage and the second one through the direct current excitation of the tactile interface. Another technology is based on friction reduction using ultrasonic vibrations via the "squeeze film effect" [1316]. In the remainder of this paper, we are particularly interested in the latter technology. 
It has been shown that programmable friction feedback on tactile displays improves performances on different interaction tasks as in [17-20]. Therefore, several studies and guidelines have been proposed in $[21,22]$ to assist designers to find out how users feel and manipulate tactile elements on haptic feedback displays for touch interactions. On the other hand, Serafin et al. [23] has investigated friction-based musical instruments. Nevertheless, there is still no studies as far as we aware for the explanation of how the musical interaction on tactile displays can be enriched with programmable friction and haptic feedback.

In this context, we discuss how haptic feedback by means of programmable friction on tactile interfaces might influence and enrich musical interaction. In particular, we explore different mappings between sound synthesis parameters and haptic feedback, and evaluate the impact of these mappings on user experience when performing a given musical task.

\section{Previous Studies on Musical Interactions with Haptic Interfaces}

In $[24,25]$ the authors show that there is a close relationship between haptic feedback and sound production in computer music performances. In [2] the authors design a new audio-haptic interface on mobile phones. The creation of a mobile music player accompanied with synchronized haptic feedback to create a novel method of audio playback on a mobile device is described in [3]. In [5] a cross-modal interface of digital audio to the haptic domain for visually impaired sound producers is presented. The design of a hardware/software system for rendering multi-point, localized vibrotactile feedback in a multi-touch musical interface is described in [4]. A new approach to the integration of vibrotactile feedback into digital musical instrument designs is investigated in [6]. In [8] two tactile interfaces for the granular synthesis technique is proposed. Besides, in [7] a touchscreen interface for live performance with real-time granular synthesis is studied.

However, there is still no investigation regarding the potential influence of tactile feedback displays with programmable friction on musical interactions.

\section{Exploring Mappings between Sound and Haptic Rendering}

In this section we discuss mapping opportunities for tactile feedback with programmable friction. Notice that, due to the nature of the technology used for the tactile feedback, haptic feedback occurs only when sliding the finger on the surface. Instruments than can be built with this technology are therefore similar to acoustic friction-based instruments [23]. Therefore musical gesture which can be augmented correspond to fingers displacements on the surface, i.e. not tapping. Usable musical gesture parameters, which can be mapped to sound parameters, therefore include speed, curvature, shape, direction and so on. 
We propose to classify feedback according to its relation with both sound parameters and gestural parameters. We define four categories, labelled C1 to C4.

\begin{tabular}{|c|c|c|}
\hline & Distinct from audio & Linked to audio \\
\hline Distinct from gesture & C1 & C2 \\
\hline Linked to gesture & C4 & C3 \\
\hline
\end{tabular}

Table 1: Classification of tactile feedback with programmable friction

In category $\mathrm{C} 1$, the tactile feedback is distinct from both input gestures and audio feedback, which means it can change and provide information without changes perceived in the sound or in the gestural parameters. This can be used to provide information on current gesture to sound mappings before their results are heard, as a sort of feed-forward that guides the musician's interactions.

In category $\mathrm{C} 2$, the tactile feedback amplifies the audio feedback but is still distinct from input gestures. This can be used to provide feedback on sound parameters which are mapped to non-gestural parameters (e.g. position) when there the sound result is perceived.

In category $\mathrm{C} 3$, the tactile feedback is linked with both audio and gestures, and might amplify both of them. It can be used as in [4] to amplify both or either of the gesture and audio feedback, for example increasing self-agency of the musician with the instrument, i.e. provide a better sensation of control over the instrument.

Finally, in $\mathrm{C} 4$ the feedback is linked with the gesture but distinct from the audio, which can be used for preparation gestures. For example it may provide information on gestural parameters before reaching a zone where the gesture will actually trigger sound, allowing the musician to anticipate the sonic result of their actions.

\section{Preliminary Experiment}

We carried out an experiment to find out how the ultrasonic based haptic interface with programmable friction might influence and enrich the interaction with Digital Music Instruments (DMIs). To do so, we tested the effect of added tactile feedback, and three mappings that follow the categories $\mathrm{C} 1-\mathrm{C} 3$, in a musical phrase replication task.

\subsection{Apparatus}

We used E-ViTa (Enhanced Visual-Tactile Actuator), a haptic feedback tablet based on ultrasonic vibrations for haptic rendering [26] which is developed on a "Banana Pi", a single-board computer (Shenzhen LeMaker Technology Co. Ltd, 
China) with $1 \mathrm{GHz}$ ARM Cortex-A7, dual-core CPU and $1 \mathrm{~GB}$ of RAM working in parallel with a STM32f4 microcontroller (STMicroelectronics, France). The communication between the microcontroller and the single board computer is provided via the Serial Peripheral Interface (SPI) bus at $10 \mathrm{kHz}$. This singleboard computer is connected to a 5 inches capacitive touchscreen (Banana-LCD 5"-TS, MAREL, China) for detecting the user's finger position on the display with a sampling frequency of $60 \mathrm{~Hz}$. The piezoceramic actuators are placed on each side of the touchscreen. The microcontroller synthesizes a pulse-width modulation (PWM) signal to pilot a voltage inverter to actuate the piezoceramics. The E-ViTa tablet has a resolution of $800 * 480$ pixels. The detailed structure of $E$-ViTa haptic tablet is shown in the figure 1.
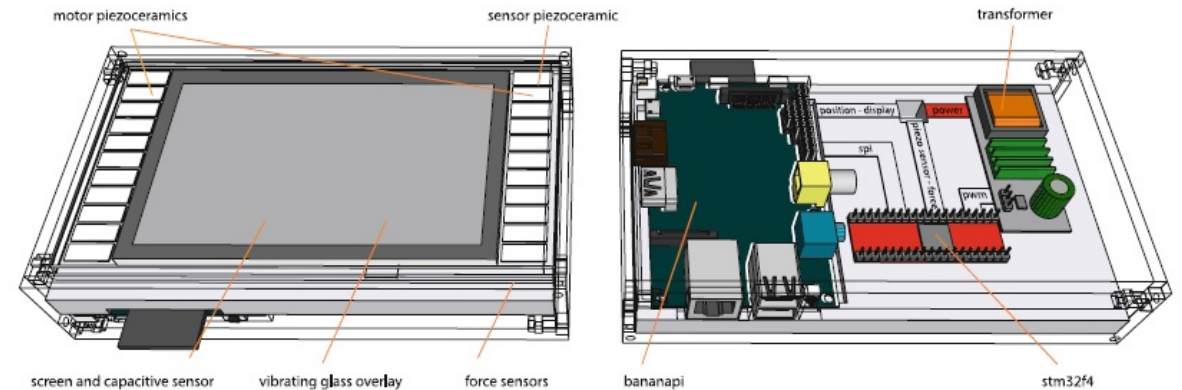

Fig. 1: The structure of $E$-ViTa haptic feedback tablet [26]

\subsection{Participants}

Six volunteers ( 4 male and 2 female) from the age of 27 to 33 with a mean age of $29.14(\mathrm{SD}=1.95)$ took part in our experiment. They were all regular users of at least one tactile display (i.e. smart-phone or tablet) during their daily life. The experiment took on average approximately 35 minutes for each of the participant. All the participants used headphones in order to prevent the influence of the little noises from the haptic tablet.

\subsection{Design}

We used a basic form of FM synthesis with only two oscillators using Pure Data to generate the auditory signals. In FM synthesis [27], the timbre of a simple waveform is changed by modulating its frequency in the audio range which leads to a more complex waveform with a different-sounding tone. In our case we have two sine waves: modulating wave and carrier wave in which the modulating wave changes the frequency of the carrier wave. The communication between the PC (which generates the FM synthesis) and the haptic feedback tablet is done 
using the OpenSoundControl protocol. We have to note that in the E-ViTa haptic feedback tablet, the generated tactile feedback (with a specific spatial frequency and amplitude) is always proportional to the user's finger velocity as explained in [26]. In other words, there is a linear transfer function between the tactile signal's spatial frequency $(\mu m)$ and the sound signal frequency $(H z)$ which is proportional to the user's finger velocity $(\mathrm{mm} / \mathrm{s})$ at each moment. This relationship can be expressed as the following equation:

$$
\text { Sound Signal Frequency }(H z)=\frac{\text { Tactile Signal Spatial Frequency }(\mu \mathrm{m})}{\text { Finger Velocity }(\mathrm{mm} / \mathrm{s})}
$$

In all of the three tested mappings, the volume of the sound is mapped to the speed of the gesture, i.e. the speed at which the user's finger moves on the surface, and the pitch of the sound is mapped to the $\mathrm{Y}$ axis of the tablet. We then defined 3 different mappings between the auditory and tactile signals as below:

- Mapping 1: In the first mapping, the tactile signal is associated to the resonance of the produced sound, i.e. the time it takes for the sound to fade out. The more resonance there is the less friction can be felt. However this parameter is only heard when the user's gesture stops. The resonance parameter is mapped to the $\mathrm{X}$ axis on the tablet. This mapping corresponds to category $\mathrm{C} 1$.

- Mapping 2: In the second mapping, the tactile signal is associated to the roughness of the sound, which is produced by modifying the modulation amplitude of the FM synthesis. The higher the amplitude of the modulation is, the rougher the sound is, and the more friction felt by finger. This parameter is mapped to the $\mathrm{X}$ axis of the tablet. This mapping corresponds to category C2.

- Mapping 3: In the third mapping, the friction is only mapped to the gesture speed and therefore to the volume of the sound signal. This mapping corresponds to category C3.

\subsection{Task and Procedure}

First of all a brief description of our task as well as all the necessary instructions for interacting with our audio/haptic tactile interface were given to each participant. We asked the participants to do a replication task of previously recorded sounds with a duration of a few seconds for each of the 3 provided mappings. In order to prevent any influence on the participants' performances of the given task, the order of the three tested mappings was randomized. The participants were free to explore the surface as long as they wanted and then replicated the provided sounds. We also recorded the gesture trajectory of finger movements of each participant for the further analysis in our study. The E-ViTa haptic display used in our experiment is illustrated in figure 2 


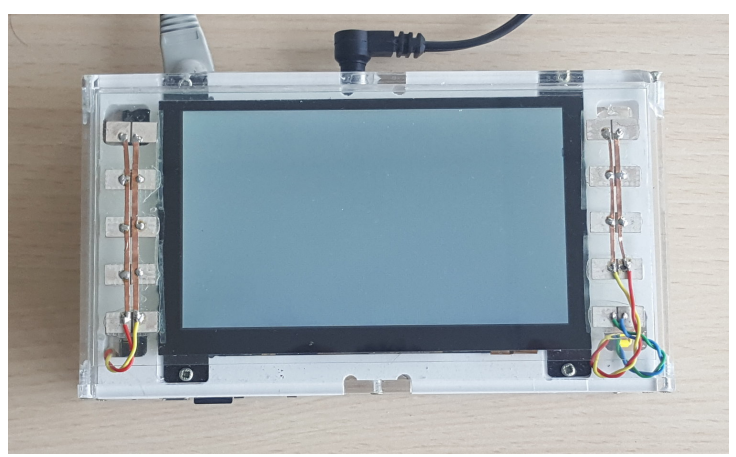

Fig. 2: The E-ViTa haptic feedback display used in our experiment

\section{Results and Discussion}

We used qualitative evaluation methods previously discussed to evaluate DMIs and musical performances in [28,29].

We designed our questionnaire in order to avoid influencing the participant's answers. Thus, we asked the following questions to the participants: 1) How do you feel about the sound you created? 2) How do you describe your experiment with our audio/haptic interface? 3) Can you identify and distinguish each of the 3 mappings? 4) How do the 3 mappings compare and which one did you prefer? 5) Do you consider haptic feedback as a useful tool for sound synthesis and musical performances?

In summary, all the participants declared that the audio/haptic interface is very useful and interesting to enrich their musical perception. In particular, they expressed that the provided friction-based haptic feedback allowed them to feel the interaction with the real instruments as well as feeling what they hear simultaneously. They were all able to correctly identify and distinguish the three provided mappings. This means that they were all capable to detect which sound parameters in the 3 cases were associated to the corresponding tactile feedback.

$50 \%$ of the participants preferred mapping 1 in which the tactile signal was mapped to the resonance of the sound. The remaining $50 \%$ of the participants preferred mapping 3, in which the generated sound was correlated with the gesture speed. This does make sense, taking into account that by principle; our audio/haptic interface exploit user's gesture velocity for tactile feedback rendering.

We also analyzed the gesture trajectories for each of the 3 provided mappings in order to study the influence of tactile feedback for users' performances of each musical gesture. The trajectories of participants when replicating a reference sound, shown in figures 3, 4 and 5, suggest that the tactile feedback has an effect on their gestures, as some variations can be seen. However, we still need further investigations and experiments to better evaluate the tactile feedback effect on musical gestures, with more participants and refined trajctory analysis. 
In all the following figures, $x$ and $y$ correspond to the $\mathrm{X}$ and $\mathrm{Y}$ axes of the haptic tablet with the resolution of $800 * 480$ pixels.

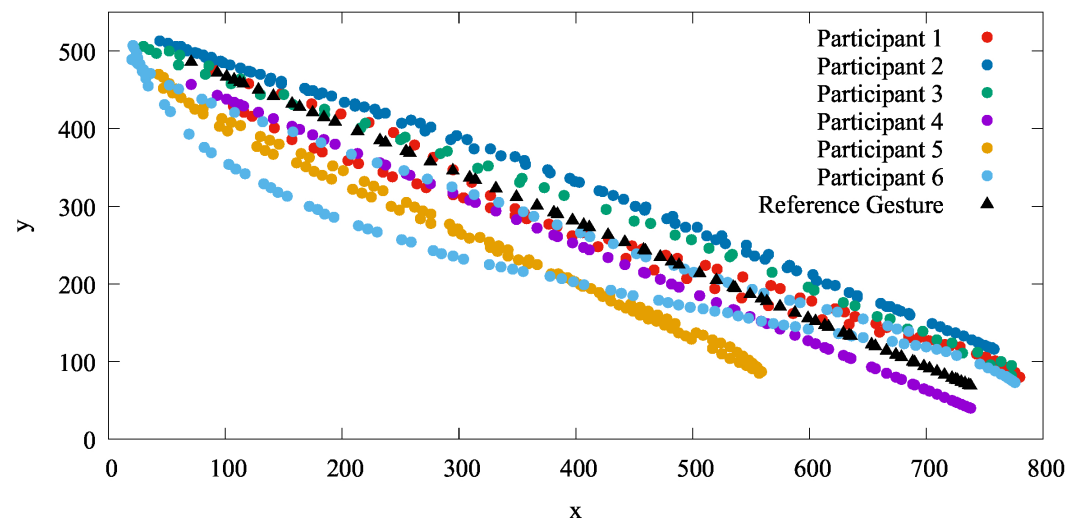

(a) No feedback

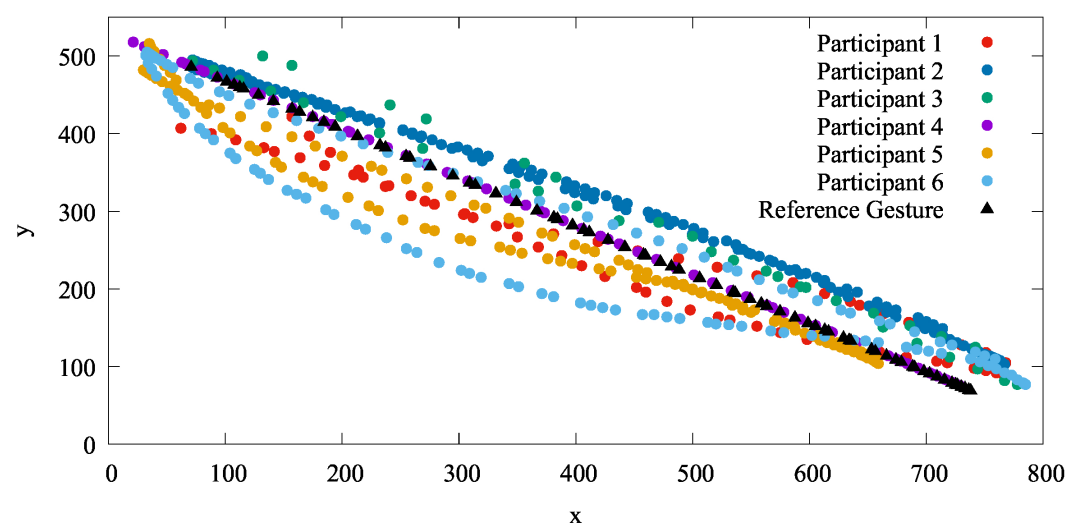

(b) Tactile feedback

Fig. 3: The gesture trajectories of mapping 1

Some of the participants' comments are as follow: "I think that it's a very enjoyable and interesting experience". "I feel that I'm playing a real string musical instrument (such as a guitar), taking into account the haptic feedback and the various type of sounds that I'm able to create." Or, "I have never played a DMI before, however this interface may considerably facilitate the process of learning the sound synthesis and musical productions for me. The haptic feedback also help me to have a better feeling of the sounds that I create." Also, "As a musician I believe this audio/haptic tactile display, enables us to enhance our perception of the basic principles of theory and harmony in music productions. It 


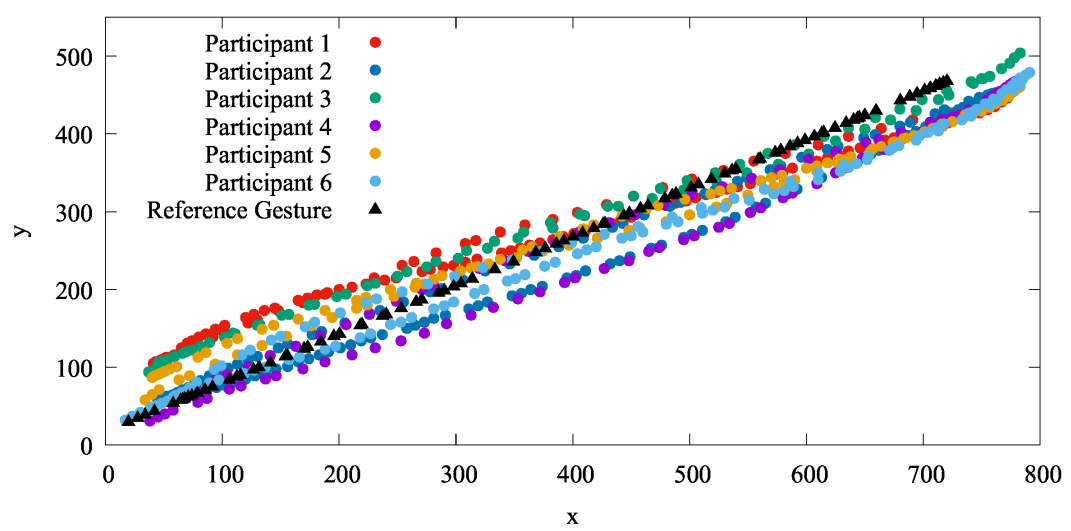

(a) No feedback

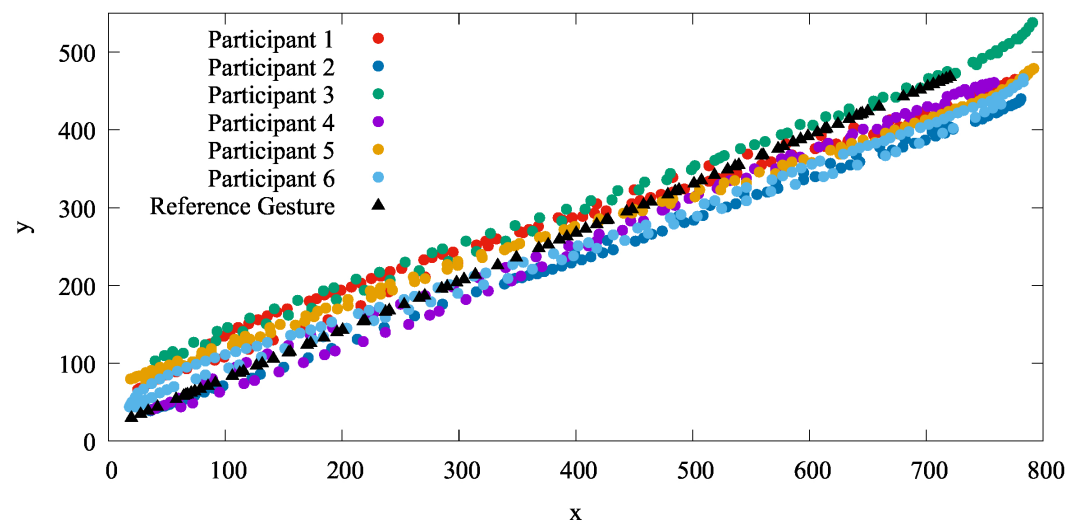

(b) Tactile feedback

Fig. 4: The gesture trajectories of mapping 2

may also be useful to teach basic musical performances to beginner users as they are capable of hearing and feeling the sounds simultaneously." Or "The provided haptic feedback permits me to perform the appropriate gesture faster and easier for each mapping (specially for mapping 3), even without looking at the device." Or "The tactile feedback gives me an extra dimension to the music which I have never experienced before. In fact it provides a novel sensational feeling to the music that I used to only hear it."

\section{Conclusion and Future Work}

In the present paper we have reported our preliminary investigations regarding to the potential influences of tactile feedback displays with programmable friction on users' musical interactions by means of a qualitative approach. We have 


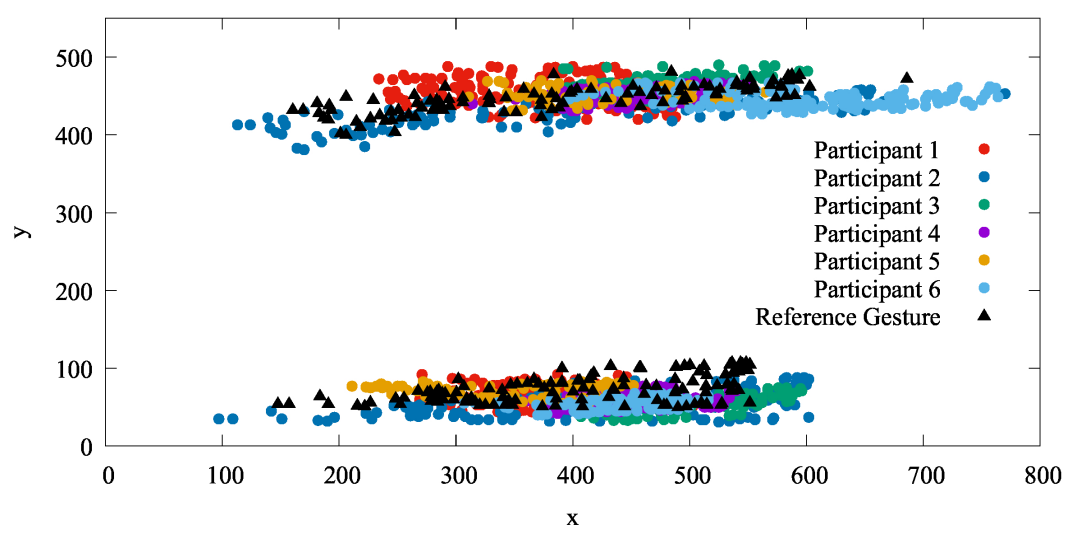

(a) No feedback

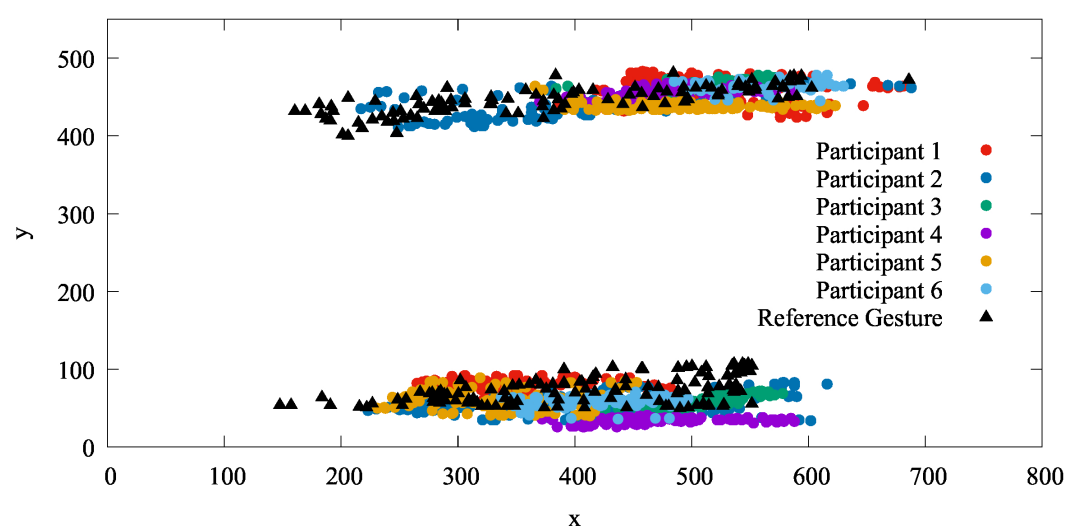

(b) Tactile feedback

Fig. 5: The gesture trajectories of mapping 3

proposed four categories of mappings between the sound parameters and tactile feedback and analyzed user's experiences with three of them. Our preliminary results suggest that all the users consider the friction-based tactile feedback as a useful and interesting phenomenon for enhancing musical interactions, performances and learning. As future work, we aim to investigate the tapping gesture to our audio/haptic interface which could allow us to simulate a wide range of instruments (e.g. touching the piano keyboard).

\section{Acknowledgements}

The authors would like to appreciate Babak Rabbanipour for his useful help in our study. This work is partially funded by European ERDF grants (IRCICA, CPER MAUVE) and ANR funding agency (equipex IRDIVE). 


\section{References}

1. Buxton, W., Hill, R., Rowley, P.: Issues and Techniques in Touch-sensitive Tablet Input. In: SIGGRAPH '85, 12th Annual Conference on Computer Graphics and Interactive Technique, ACM, (1985)

2. Chang, A., O'Sullivan, C.: Audio-haptic Feedback in Mobile Phones. In: CHI '05 Extended Abstracts on Human Factors in Computing Systems, ACM, (2005)

3. Baillie, L., Beattie, D., Morton, L.: Feel What You Hear: Haptic Feedback As an Accompaniment to Mobile Music Playback In: IwS '11 Interacting with Sound Workshop: Exploring Context-Aware, Local and Social Audio Applications, ACM, (2011)

4. Papetti, S., Schiesser, S., Fröhlich, M.: Multi-Point Vibrotactile Feedback for an Expressive Musical Interface. In: NIME '15, New Interfaces for Musical Expression, Springer, (2015)

5. Tanaka, A., Parkinson, A.: Haptic Wave: A Cross-Modal Interface for Visually Impaired Audio Producers. In : CHI '16, Conference on Human Factors in Computing Systems, ACM, (2016)

6. Birnbaum, D., Wanderley, M.M: A Systematic Approach to Musical vibrotactile feedback. In: ICMC'07, International Computer Music Conference, (2007)

7. Sanganeria, M., Werner, K.J.: GrainProc: a real-time granular synthesis interface for live performance. NIME '13, New Interfaces for Musical Expression, Springer, (2013)-

8. O'Modhrain, S., Essl, G.: PebbleBox and CrumbleBag: Tactile Interfaces for Granular Synthesis. In: NIME '04, New Interfaces for Musical Expression, Springer, (2004)

9. Choi, S. and Kuchenbecker, K.J.: Vibrotactile Display: Perception, Technology and Applications. IEEE 2013, Volume 101, No. 9, (2013)

10. Bau, O., Poupyrev, I., Israr, A. and Harrison Ch.: TeslaTouch: Electrovibration for Touch Surfaces, In: UIST 2010, ACM, (2010)

11. Senseg Inc., http://senseg.com

12. Shultz, C.D, Peshkin, M.A, Colgate, J.E.: Surface haptics via electroadhesion: Expanding electrovibration with Johnsen and Rahbek. In: WHC 2015, IEEE, (2015)

13. Amberg, M., Giraud, F., Semail, B., Olivo, P., Casiez, G., and Roussel, N. : STIMTAC: A Tactile Input Device with Programmable Friction., In: UIST 2011, ACM, (2011)

14. Ben Messaoud, W., Amberg, M., Lemaire-Semail, B., Giraud, F. and Bueno, M.A.: High fidelity closed loop controlled friction in SMARTTAC tactile stimulator. In: 17th European Conference on Power Electronics and Applications ,EPE'15 ECCEEurope, (2015)

15. Giraud, F., Amberg, M., Lemaire-Semail, B. and Casiez, G.: Design of a transparent tactile stimulator. In: IEEE Haptics Symposium (HAPTICS'12), (2012)

16. Winfield, L., Glassmire, J., Colgate, J.E., and Peshkin, M: T-PaD: Tactile Pattern Display Through Variable Friction Reduction, In: WHC 2007, IEEE, (2007)

17. Levesque, V., Oram, L., MacLean,K., Cockburn, A., Marchuk N.D. , Johnson,D, Colgate, J.E, Peshkin, M.A.: Enhancing Physicality in Touch Interaction with Programmable Friction. In: CHI 2011, ACM, (2011)

18. Casiez, G., Roussel, N., Vanbelleghem, R., Giraud, F.: Surfpad: Riding Towards Targets on a Squeeze Film Effect. In: CHI 2011, ACM, (2011)

19. Zhang, Y. and Harrison, Ch.: Quantifying the Targeting Performance Benefit of Electrostatic Haptic Feedback on Touchscreens. In: ITS '15, International Conference on Interactive Tabletops \& Surfaces, ACM, 2015 
20. Israr, A., Bau, O., Kim, S.C., Poupyrev, I.: Tactile feedback on flat surfaces for the visually impaired. In: CHI EA, ACM, (2012)

21. MacLean, K.E.: Haptic interaction design for everyday interfaces. Reviews of Human Factors and Ergonomics Society, (2008)

22. Kalantari, F., Grisoni, L., Giraud, F. and Rekik, Y.: Finding the Minimum Perceivable Size of a Tactile Element on an Ultrasonic Based Haptic Tablet., In: ISS'16 Interactive Surfaces and Spaces, ACM, (2016)

23. Serafin, S., Young, D.: Toward a Generalized Friction Controller: from the Bowed String to Unusual Musical Instruments. In: NIME '04, International Conference on New Interfaces for Musical Expression, (2004)

24. Chu, L.L.: Haptic feedback in computer music performance. In: ICMC'96, International Computer Music Conference, (1996)

25. Chafe, C.: Tactile audio feedback. In: ICMC'93, International Computer Music Conference, (1993)

26. Vezzoli, E., Sednaoui, T., Amberg, M., Giraud, F. and Lemaire-Semail, B.: Texture Rendering Strategies with a High Fidelity - Capacitive Visual-Haptic Friction Control Device. Haptics: Perception, Devices, Control, and Applications, (2016)

27. Chowning, J.M., Bristow, D.: Fm Theory and Applications: By Musicians for Musicians. Hal Leonard Corp, (1987)

28. Ghamsari, M., Pras, A., Wanderley, M.M.: Combining musical tasks and improvisation in evaluating novel Digital Musical Instruments. In: CMMR '13, International Symposium on Computer Music Multidisciplinary Research, Springer, (2013)

29. Barbosa, J., Malloch, J., Wanderley, M.M, Huot, S.: What does âĂIJEvaluationâĂ mean for the NIME community? In: NIME '15, New Interfaces for Musical Expression, Springer, (2015) 